\title{
APPLICATION OF DIMENSION REDUCTION METHODS TO DOW JONES INDUSTRIAL AVERAGE
}

In-Jae Kim, Minnesota State University, Mankato, MN, U.S.A. Dooyoung Shin, Minnesota State University, Mankato, MN, U.S.A. Kwang-il Choe, Minnesota State University, Mankato, MN, U.S.A.

dx.doi.org/10.18374/JABE-19-3.4

\begin{abstract}
In this paper we use Principal Component Analysis (PCA) to show that the dimension of the Dow sample data (which is 30) can be reduced to be three (i.e., three dominant business sectors), while capturing the most of its total variance. Using PCA we also conclude that during the time period of the sample data, the stock prices included in the Dow were in the trend of increasing. In addition to PCA, we apply factor analysis to identify the three business sectors which had the strongest presence during the time period: Health Care, Individual Consumer Credit \& Goods, and Information Technology Intensive Business. Furthermore, we show that the individual consumer credit \& goods sector had the highest growth rate during the time period of the sample data.
\end{abstract}

Keywords: Stock Market, Dow Jones Industrial Average, Dimension Reduction, Principal Component Analysis, Factor Analysis 